\title{
Hypoxic Preconditioning of the Skim Graft oonor Area: Clinical and Morphological Assessment
}

DOI: $10.17691 / \mathrm{stm} 2018 \cdot 10.3 .13$

Received February 10,2018,

N.Yu. Orlinskaya, MD, DSc, Professor, Head of the Department of Pathology and Tissue Preservation, Institute of Traumatology and Orthopedics';

D.V. Davydenko, PhD, Researcher, Department of Pathology and Tissue Preservation,

Institute of Traumatology and Orthopedics';

M.V. Bagryantsev, MD, Surgeon';

M.G. Ryabkov, MD, DSC, Surgeon-Consultant2;

V.V. Kichin, MD, Surgeon';

1.V. Pavlenko, MD, Surgeon';

V.V. Beschastnov, MD, DSc, Surgeon-Consultant ${ }^{2}$

Privolzhsky Research Medical University, 10/1 Minin and Pozharsky Square, Nizhny Novgorod, 603005, Russia;

${ }^{2}$ Municipal Clinical Hospital No.30, 85a Berezovskaya St., Nizhny Novgorod, 603157, Russia

The aim of the study was to evaluate the method of hypoxic preconditioning of the donor area using clinical and morphological assessment of the autologous dermal transplant (skin graft).

Materials and Methods. The transplants were prepared from the skin donor area after hypoxic preconditioning. Reparative regeneration, skin graft angiogenesis and the cosmetic status of the donor area wound upon healing were studied. To this end, five patients with diabetes-associated soft tissue wounds underwent classic free skin plastic surgery (control group), and five patients underwent the similar operation preceded by hypoxic preconditioning of the donor zone (study group). On day 5 after the surgery, morphological and immunohistochemical examination of the skin transplant was performed; on day 14, the area of engraftment was evaluated.

Results. On day 14, the area of engraftment of the autologous transplant in the control group was 56 [52; 64]\%, and in the study group - 91 [84; 95$] \%$ ( $p=0.007)$. The healing of the donor area wounds in the control group was detected on day 18 [16; 21], in the study group — on day $14[12 ; 17](p=0.002)$. In the intravital histological staining of the biopsy specimen, cell-enriched skin fragments were found in the study group, whereas only cell-depleted skin fragments were observed in the control group. Upon the double sequential immunohistochemical staining with antibodies to Ki-67 and CD31 antigens, increased proliferation of endothelial cells of the dermis was noted in the study group, with the proliferation index of 29 [22; 33] versus $17[13 ; 24] \%$ in the control group $(p=0.011)$. The increased levels of antibodies to VEGF and Fli-1 in the study group also pointed to the active angiogenesis in these patients.

Conclusion. The results of the intravital histological and immunohistochemical study indicate that hypoxic preconditioning of the donor area can serve an effective tool of increasing the reparative regeneration and angiogenesis in the skin graft.

Key words: free skin plastic surgery; autologous skin graft; hypoxic preconditioning; angiogenesis; reparative regeneration.

\section{Introduction}

One of the main causes of chronic wounds of soft tissues in diabetes is tissue hypoxia developing on the background of vascular lesions in the lower limbs [1]. The diabetic foot syndrome develops in $8-10 \%$ of such patients, and, according to $\mathrm{WHO}$, the number of patients with diabetes increased 4-fold in the period from 1980 to 2014 [2]. Today's means of endovascular surgery allow surgeons to avoid limb amputation during critical ischemia [3]; however, chronic wounds sustain for quite a while, which necessitates closing the wound defect under conditions of tissue hypoxia. The problem is complicated by hyperglycemia and insufficient microcirculation in the wound zone due to glycoproteins accumulation in the arteriole basal membrane leading to increased capillary wall thickens and resulting limitations of oxygen diffusion [4].

An effective way to close such chronic wounds is free skin plastic surgery using a split flap [5]. It is known that successful engrafting of an autologous transplant requires active angiogenesis [6-8]. Under the normal conditions, a skin graft placed in the recipient wound is nutrient-supplied by diffusion and the early signs of vascularization are observed on day 5 after surgery [9]. To adapt the transplant to the conditions of oxygen deficiency and increase the chances of its survival under hypoxia, several methods of hypoxic preconditioning of the flap donor area have been proposed [10]. The principle of hypoxic preconditioning is to decrease the

Corresponding author: Maxim V. Bagryantsev, e-mail: maks-bagryancev@mail.ru 
level of microcirculation in the donor area and thereby imitate tissue hypoxia in the wound defect zone, while blood flow is monitored with laser Doppler flowmetry or tissue oximetry.

Earlier [11], we used an enzyme immunoassay to demonstrate that hypoxic preconditioning (reducing microcirculation in the skin of the donor area by applying two parallel cuts of the skin and subcutaneous tissue to the depth of the surface fascia) led to an increase in the concentrations of the specific cytokine named the hypoxia-inducible factor (HIF-1 $\alpha$ ). This factor accumulates in tissues under hypoxic conditions and stimulates the expression of proteins that regulate oxygen delivery and angiogenesis. In addition, other results [12] suggest that the accumulation of the cytokine HIF-1 $\alpha$ in the skin of the donor site may activate the process of angiogenesis in a skin graft taken from this area. No direct evidence of changes in the angiogenesis activity or reparative regeneration in the skin graft after its hypoxic training was found in the available literature.

The aim of the study was to evaluate the results of hypoxic preconditioning of the donor area using clinical and morphological assessment of the skin graft.

\section{Materials and Methods}

The clinical study was approved by the Ethics Committee for Scientific Research in Human Subjects at the Privolzhsky Research Medical University and carried out in full compliance with the current regulatory and legal acts.

The inclusion criteria for the study were:

voluntary informed consent of a patient to participate in the study;

neuro-ischemic form of the diabetic foot syndrome (stage II ) by F.M. Wagner;

reconstructive vascular surgery on the lower limb arteries performed no earlier than 14 days prior to the patient enrollment in the study;

the existence of the main blood flow in the lower limb arteries confirmed by ultrasound Doppler;

the state of the recipient wound surface, estimated at 16-17 score according to the Recipient Wound Readiness for Free Autodermoplasty (I.I. Dzhanelidze Research Institute of Emergency Medicine, Russia).

The present study included 10 patients ( 7 females and 3 males) with chronic soft tissue wounds resulted from purulent-necrotic complications of the diabetic foot syndrome. All patients were undergoing treatment in the Department of Purulent Surgery of the City Clinical Hospital No.30 of Nizhny Novgorod in 2016-2017. The patients' age varied from 55 to 85 years and it averaged at $63.2 \pm 8.1$ years. All patients suffered from type 2 diabetes mellitus. The patients were divided into two groups: the study group $(n=5)-$ with hypoxic preconditioning of the skin donor zone and control $(n=5)-$ with free skin plastic surgery in its classical version.
The preparation of the soft tissue defect (the recipient wound) for the upcoming surgery was carried out similarly in both groups. This included a daily change of polyethylene oxide ointment dressings, the mechanical removal of residual non-viable tissues and fibrin, the application of bandages with proteolytic enzymes (chymotrypsin) for two days before surgery. In patients of the study group, the donor area (anterolateral aspect of the thigh) was preliminarily subjected to hypoxic preconditioning according to Beschastnov et al. [11]. Thus, under local anesthesia, two parallel skin incisions were made to the depth of the surface fascia; the incisions were $10 \mathrm{~cm}$ long each and located at a distance of $5 \mathrm{~cm}$ from each other. Under the guidance of laser Doppler flowmetry, a skin flap was mobilized so to reduce the microcirculation rate by $50 \%$; then an intradermal cosmetic suture (Prolene 4.0) was placed.

We then studied the chronic soft tissue wound following its closure by the free skin plastic surgery. We also studied the processes of reparative regeneration and angiogenesis in the skin graft formed from the donor area (either subjected or not subjected to presurgery hypoxic preconditioning) as well as the cosmetic aspects of the donor wound healing. The proposed hypothesis was that the skin graft formed from the donor area with preliminary hypoxic preconditioning would have a larger engrafting area due to the earlier release of proangiogenic factors. The following parameters were used to evaluate this hypothesis: the relative engrafting area of the transplant, the expression of the $\mathrm{Ki}-67$ protein - the index of cell proliferation (this protein is expressed in all phases of the cell cycle except for the synthetic G0 phase), the concentrations of the proangiogenic factors CD31, Fli-1 and the level of vascular endothelial growth factor (VEGF).

In all patients, a split skin flap was excised using an electrodermatome; then the would-be transplant was placed in saline containing $4 \mathrm{mg}$ of dexamethasone for $2 \mathrm{~min}$, after which it was perforated and placed on the recipient wound. To facilitate the donor area wound healing, a dressing containing a bioplastic material based on hyaluronic acid and collagen (G-Derm, Russia) was used in patients of the study group, whereas in the control group, the donor area wound was covered with a sterile gauze pad impregnated with $5 \%$ solution of potassium permanganate.

On day 3 after the surgery, the first dressing change was performed on the recipient wound. Using vascular scissors, a viable patch (bioptate) measuring $5 \times 5 \mathrm{~mm}$ was excised from the distal edge of the graft. The sample was fixed in neutral $10 \%$ formalin. Standard histological processing was performed using an Excelsior ES machine (Thermo Scientific, USA). After the processing, paraffin blocks were prepared using a HistoStar filling station (Thermo Scientific, USA). Sections 4-6 $\mu \mathrm{m}$ thick were obtained from a Microm HM 325 microtome (Thermo Scientific, USA). The sections were stained with hematoxylin and eosin. 
Double sequential immunohistochemical staining was performed in an automated BOND-MAX module using a Bond polymer refine detection system (protocol F) and a Bond polymer refine red detection system (protocol J) (Leica Biosystems, UK). Monoclonal antibodies to Ki-67 - clone SP6 (Lab Vision Corporation, USA), to CD31 antigen - clone JC70A (Lab Vision Corporation, USA), to Fli-1 - clone MRQ-1 (Cell Marque, USA) and antibodies to VEGF-polyclonal (Diagnostic BioSystems, USA) were used.

To evaluate the cell proliferation, the number of $\mathrm{Ki}$ 67 positive cells in the dermal transplant (10 visual fields) was counted using $\times 400$ microscopy. The reaction between the $\mathrm{Ki}-67$ protein and the respective monoclonal antibodies allows one to visualize the newly divided cells, since the protein is expressed only in the nuclei of proliferating cells and can be detected in $\mathrm{G} 1$ (at the end of the phase), G2, S, and M phases of the cell cycle with maximum expression in the $\mathrm{G} 2$ and $\mathrm{M}$ phases [13]. The proliferation index (PI) was determined by the formula

$$
\mathrm{PI}=(n / N) \cdot 100 \%,
$$

where $n$ is the number of labeled nuclei; $N$ is the total number of nuclei per visual field of the microscope.

Planimetric assessment of the transplant engrafting area was carried out with digital macrophotography of the recipient wound area. The surface area of the autologous dermal transplant was calculated from the photographs using the ImageJ program.

To analyze the results of the plastic surgery procedure, we used the $D$ index, which indicated the area of successful engrafting. The $D$ was calculated from the formula

$$
D=S_{1} / S_{2} \cdot 100 \% \text {, }
$$

where $S_{1}$ was the area $\left(\mathrm{cm}^{2}\right)$ of the transplant surface immediately after its transplantation onto the recipient wound, and $S_{2}$ was the area $\left(\mathrm{cm}^{2}\right)$ of the transplant on day14 after the surgery.

For statistical data processing, the computer program Statistica 6.0 was used. To assess the significance of the differences between the compared groups, we used either the Fischer's precise test (for the qualitative comparison) or the Mann-Whitney U criterion (for the quantitative comparison). The selected parameters presented below are denoted as follows: $\mathrm{Me}$ - the median, $Q_{1}$ - the upper quartile, $Q_{3}$ - the lower quartile, $\mathrm{n}$ - the volume of the analyzed subgroup, $p-$ the value of the significance of the differences. The critical significance level was taken $5 \%(p \leq 0.05)$.

\section{Results}

Following the surgical treatment of chronic soft tissue wounds using autologous skin transplants, the $D$ index (measure of engraftment) in patients of the study group was 91 [84; 95] versus $56[52 ; 64] \%$ in the control group $(p=0.007)$.

In the control group, the early signs of healing in the donor area wounds were detected on day 18 [16; 21]; along with that, some patients complained of discomfort caused by bandage drying and sticking to the wounds; in addition, ulceration and coarse scar formation were noted. Contrary to that, in all patients of the study group, uncomplicated healing of the donor area wounds was found on day $14[12 ; 17]$ upon surgery $(p=0.002$ when compared to control); the G-Derm bandages caused less discomfort in these patients (Figure $1(a)$ ). In the study group, the cosmetic look of the donor area wound after treatment was given the "good" mark by all patients. In the remote postoperative period, a soft, elastic, mobile skin fragment of body color was formed (Figure 1 (b)).

The histological picture of the skin graft samples from the study group was a cell-enriched fragment of the
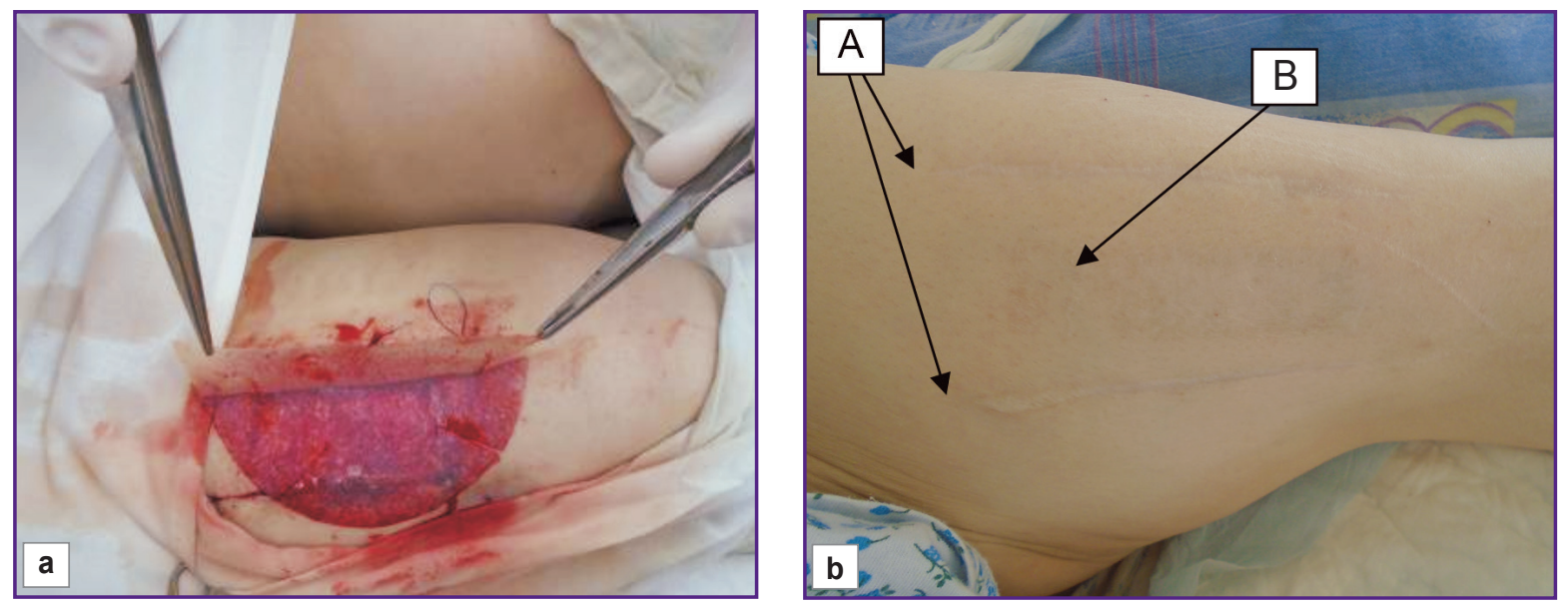

Figure 1. Healing of the donor area wound in patient E.:

(a) coating with the G-Derm bioplastic material after hypoxic preconditioning; (b) the wound appearance after 1 year: $A$ - scars left of two parallel incisions; $B$ - the donor wound zone with scar formation 

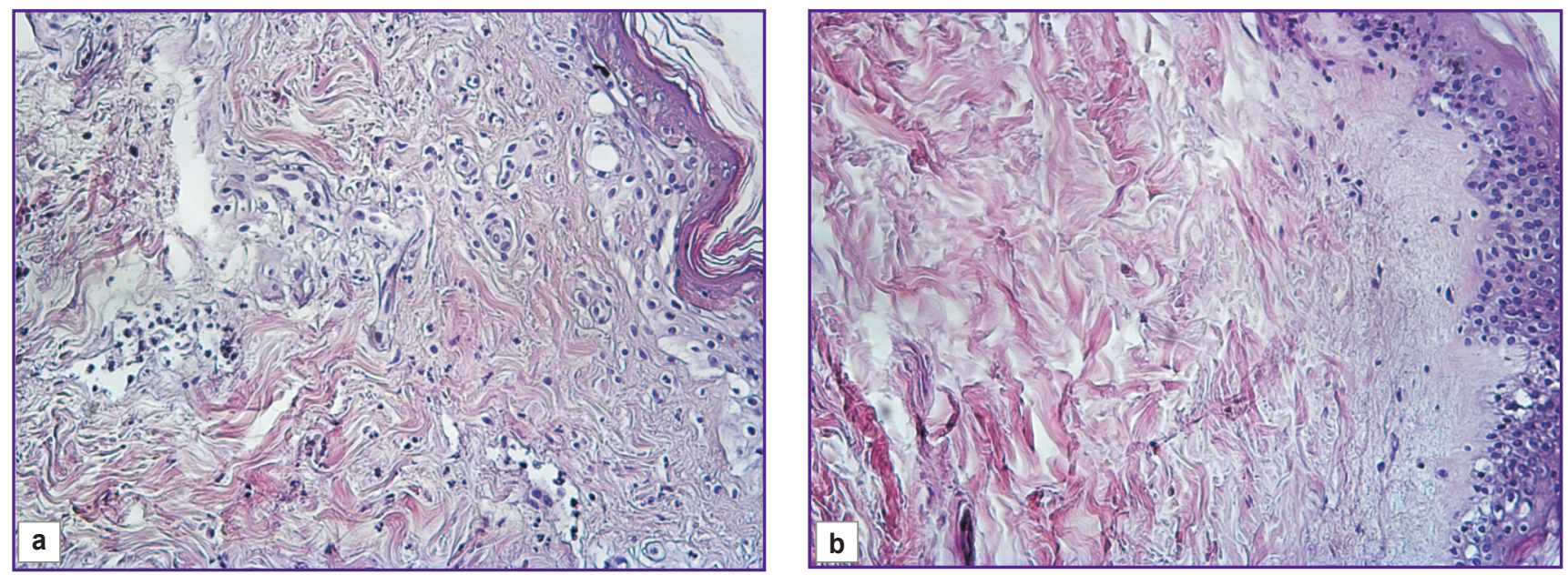

Figure 2. Microphotographs of autologous dermal transplants in the study (a) and control (b) groups; staining with hematoxylin and eosin; $\times 200$
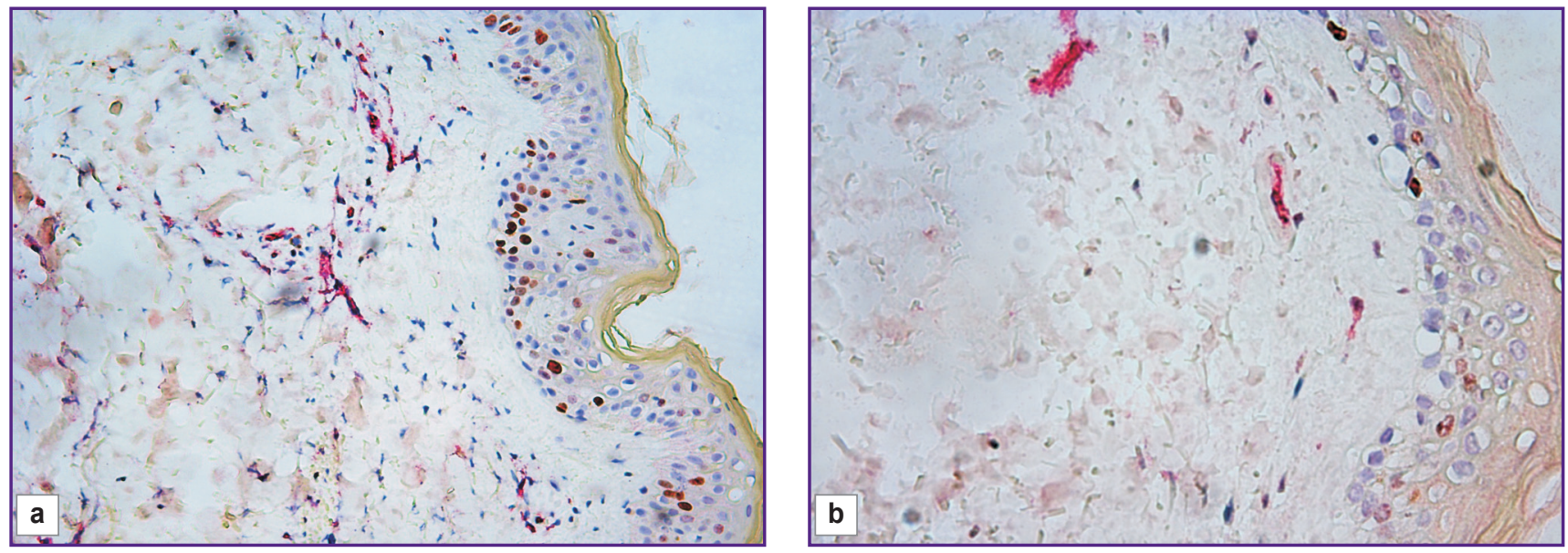

Figure 3. Histology of transplants in the study (a) and control (b) groups:

proliferation of dermis cells, Ki-67-positive cells (brown); membranes of endothelial cells (red); double immunohistochemical staining with antibodies to Ki-67 and CD31 antigens; $\times 200$

skin, with plenty of blood vessels of the endothelial type (Figure 2 (a)). In samples from the control group, celldepleted areas could be seen (Figure 2 (b)).

During engraftment, the formation of capillaries and other signs of angiogenesis are expressed in the graft portion adjacent to the recipient bed. In immunohistochemical staining with antibodies to $\mathrm{Ki}-67$ and CD31, a higher proliferation rate of dermis endothelial cells was found in the study group (Figure 3 (a)) as compared to the control one (Figure $3(b)$ ).

The reticular layer of the grafts in patients of the control group contained few foci of connective tissue necrosis; in the subcutaneous fat tissue, edema and inflammation dominated. In the study group (after hypoxic preconditioning), the occurrence of erythrocyte stasis in blood vessels of the papillary layer was much less pronounced. In the preconditioned samples, there were a markedly low number of hemorrhages in the papillary and reticular layers of the dermis, a significant decrease in necrosis and necrobiosis in the graft tissue, and almost no signs of abnormal microcirculation.

According to the immunohistochemical examination of histological preparations, the $\mathrm{Pl}$ in the control group was $17[13 ; 24] \%$, and in the study group - $29[22 ; 33] \%$ $(p=0.011)$.

In the study group, transverse sections of the dermis showed a large number of cells expressing VEGF receptors (Figure $4(a)$ ), in the control group this number was minimal (Figure 4 (b)).

In the graft tissues of both groups, there were quite a few cells expressing the Fli-1 protein; in the study group, the number of Fli-1 positive endothelial cells exceeded that in the control group (Figure 5). 

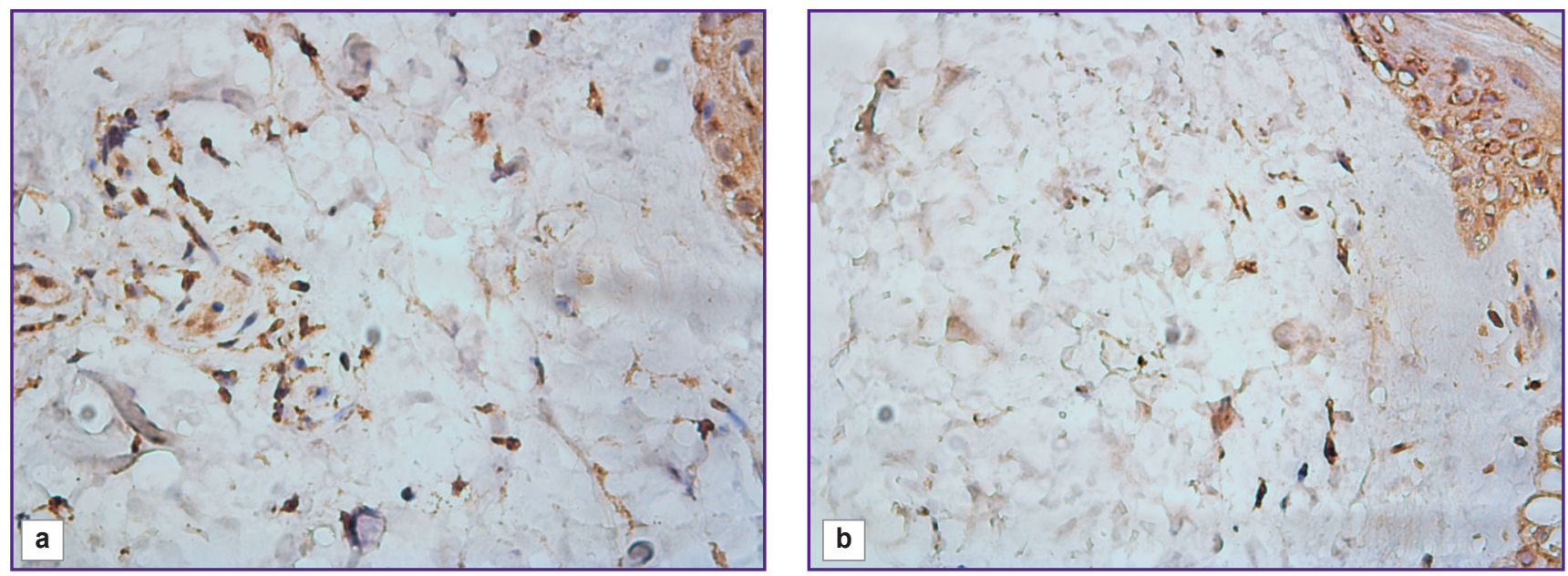

Figure 4. Microphotographs of transplants in the study (a) and control (b) groups:

cells expressing receptors to the vascular endothelial growth factor; immunohistochemical staining with antibodies to VEGF; $\times 400$
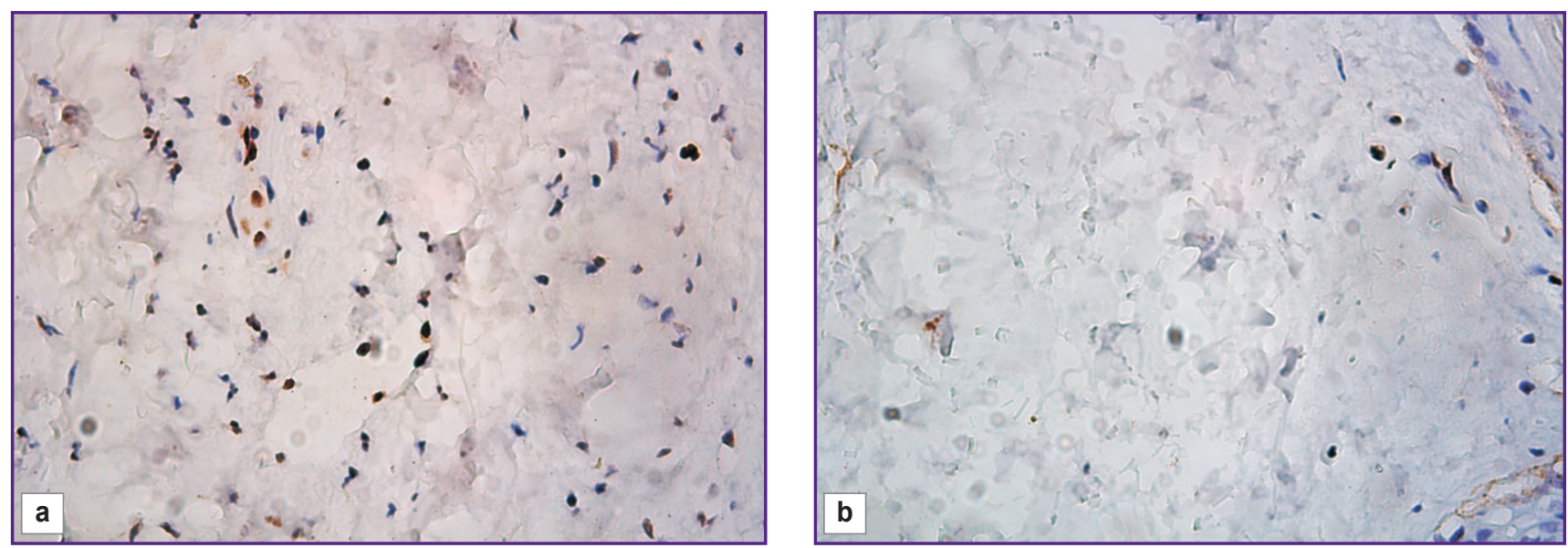

Figure 5. Microphotographs of transplants in the study (a) and control (b) groups; immunohistochemical staining with antibodies to Fli-1; $\times 200$

\section{Discussion}

Today, it is almost commonly accepted that hypoxia plays a key role in the morphogenesis of chronic wounds; as a response, the body activates its angiogenesis to provide oxygen transport to the affected tissues $[14,15]$. The growing interest in the possibilities of further enhancing the angiogenesis necessitates the development of new methods of plastic surgery including those capable of stimulating angiogenesis by hypoxic preconditioning. There is also a need in objective criteria to evaluate and assess the engraftment and treatment outcomes.

In the present study, the proliferative activity in the autologous dermal transplant was used to assess the effect of hypoxic preconditioning on the transplant engraftment and wound healing. One of the commonly used markers of skin cell proliferation is the expression of the Ki-67 protein [16]. The results obtained in our study indicate a significant increase in the proliferation activity of skin graft tissues earlier subjected to hypoxic preconditioning.

Since the proliferative activity usually correlates with the timely and complete incorporation of the graft into the systemic blood circulation, the observed increase in the number of new blood vessels came by no surprise. We were able to demonstrate this enhanced angiogenesis by using the method of double sequential immunohistochemical staining of histological slides with antibodies to Ki-67 and CD31 antigens. Specifically, the increased expression of the CD31 endothelial marker indicated an intense formation of vascular beds in the preconditioned skin grafts. Also in these grafts, an increase in the expression of VEGF - the main angiogenic factor - was found.

In summary, the method of preliminary hypoxic 
preconditioning of an autologous skin transplant is a promising way to improve the outcome of free skin plastic surgery as evidenced by the presented immunohistochemical results.

\section{Conclusion}

Hypoxic preconditioning of the skin donor area prior to free plastic surgery with a split skin flap stimulates angiogenesis and reparative regeneration in the transplanted skin tissue, and also increases the area of its engraftment from $56[52 ; 64]$ to $91[84 ; 95] \%$ $(p=0.007)$.

Research funding and conflict of interest. The study was not funded by any sources, and there are no conflicts of interest related to this study.

\section{References}

1. Catrina S.B., Zheng X. Disturbed hypoxic responses as a pathogenic mechanism of diabetic foot ulcers. Diabetes Metab Res Rev 2016; 32(1): 179-185, https://doi.org/10.1002/ dmrr.2742.

2. World Health Organization. Global report on diabetes: 2016. URL: http://www.who.int/diabetes/global-report/en.

3. Lipatov K.V., Permyakov S.V., Asatryan A.G., Borodin A.V., Gostishchev V.K. Endovascular surgery for critical ischemia of lower extremities with suppurative-necrotic lesion of the feet. Hirurgia. Zhurnal im. N.I. Pirogova 2017; 9: 4-16.

4. Risman B.V. Differentiated tactics of the skin postoperative defects closing in patients with pyo-necrotic complications of diabetic foot. Novosti khirurgii 2011; 19(2): 66-71.

5. Kotick J.D., Sandelin R.S., Klein R.D. Deep inferior epigastric perforator free flaps for use in complicated groin wound repair: a case report of severe groin scar contracture and review of pedicled and free flaps in groin wound repair. J Hand Microsurg 2017; 9(2): 101-106, https://doi. org/10.1055/s-0037-1604475.

6. Capla J.M., Ceradini D.J., Tepper O.M., Callaghan M.J.,
Bhatt K.A., Galiano R.D., Levine J.P., Gurtner G.C. Skin graft vascularization involves precisely regulated regression and replacement of endothelial cells through both angiogenesis and vasculogenesis. Plast Reconstr Surg 2006; 117(3): 836844, https://doi.org/10.1097/01.prs.0000201459.91559.7f.

7. Mavlikeev M.O., Titova A.A., Gudz D.O., Deev R.V. Modern methods for angiogenesis assessment in clinical practice. Nauka molodykh - Eruditio Juvenium 2017; 5(1): $110-123$.

8. Livanova A.A., Deev R.V., Rizvanov A.A. Current methods in experimental angiogenesis investigation. Geny $i$ kletki 2015; 10(1): 115-127.

9. Bogdanov S.V., Terman E.A., Bogdanov Y.A. Pathomorphological aspects of modern ways of surgical treatment in combustiology. Meditsinskiy vestnik Yuga Rossii 2016; 2: 33-38.

10. Vaschenko L.N., Dashkova I.R., Kechedzhieva E.E., Babieva S.M. The ability to combine autodermoplasty in the treatment of patients with malignant tumors of the skin extremities (clinical observation). Sovremennaya onkologiya 2015; 17(4): 45-50.

11. Beschastnov V.V., Izmajlov S.G., Ryabkov M.G., Bagryancev M.V., Shchelchkova N.A., Peretyagi P.V. Training method of split-thickness skin grafts to hypoxic conditions due to the compromised microcirculation. Vestnik eksperimentalnoy i klinicheskoy khirurgii 2017; 10(1): 72-78, https://doi. org/10.18499/2070-478x-2017-10-1-72-78.

12. Semenza G.L. Hypoxia-inducible factors in physiology and medicine. Cell 2012; 148(3): 399-408, https://doi. org/10.1016/j.cell.2012.01.021.

13. Diagnosticheskaya immunogistokhimiya opukholey [Diagnostic immunohistochemistry of tumors]. Pod. red. Gluzmana D.F. [Gluzman D.F. (editor)]. Kiev: Morion; 2003; $193 \mathrm{p}$.

14. Potente M., Gerhardt H., Carmeliet P. Basic and therapeutic aspects of angiogenesis. Cell 2011; 146(6): 873887, https://doi.org/10.1016/j.cell.2011.08.039.

15. Zimna A., Kurpisz M. Hypoxia-inducible factor-1 in physiological and pathophysiological angiogenesis: applications and therapies. Biomed Res Int 2015; 2015: 1-13, https://doi.org/10.1155/2015/549412.

16. Kirik O.V., Beznin G.V., Korzhevskiy D.E. Proliferation markers used in histological studies. Morfologiya 2009; 136(6): 95-100. 